Manuscript prepared for J. Name

with version 1.3 of the $\mathrm{LT}_{\mathrm{E}} \mathrm{X}$ class copernicus.cls.

Date: 18 May 2009

\title{
Analytical solutions for anisotropic MHD shocks
}

\author{
V. Génot ${ }^{1}$ \\ ${ }^{1}$ CESR, Université de Toulouse (UPS) \& CNRS (UMR5187), Toulouse, France \\ Correspondence to: Vincent.Genot@cesr.fr
}

\begin{abstract}
A new method to analytically solve the anisotropic MHD system of equations describing shock transitions is presented. As this system is known to be under-determined (there is more unknown parameters than available equations) free parameters must be chosen. From observational contraints it appears that the magnetic amplitude jump is a good 5 candidate as it is generally available more frequently and more precisely than other jump variables. With this approach we obtain an explicit expression for the density compression ratio for arbitrary upstream parameters and shock geometry. Downstream anisotropy and pressure are also calculated. The results are tested against an other approach and compared with observations from the Earth's bow shock and the solar wind termination shock.
\end{abstract}

\section{Introduction}

The MHD formalism describing transitions across shocks has been employed successfully in many astrophysical situations. The general goal is to predict downstream conditions from the knowledge of upstream conditions and shock geometry. The latter is characterized by the shock angle $\theta_{B n}$ between the upstream magnetic field and the shock normal. From this prediction it is possible, for instance, to get insight on the wave generation processes at work in the downstream regions of planetary bow shocks or solar wind termination shock, namely magnetosheaths or the heliosheath. Temperature anisotropy instabilities are among the most common means to generate waves. Consequently the formalism adopted must account for pressure variations in directions parallel and perpendicular to the ambiant magnetic field. 
20 Here we shall use the modified MHD Rankine-Hugoniot $(\mathrm{RH})$ relations including pressure anisotropy (Hudson, 1970). Other approaches are however possible. For instance Siewert and Fahr (2008) developed a kinetic approach which includes CGL invariance. Direct simulations in the MHD, hybrid and (recently) full kinetic formalisms are also commonly used to study shock physics but will not be discussed here; indeed we shall focus on an analytical 25 approach.

Solving the anisotropic MHD system by direct analytical means is an approach rarely investigated. Indeed this system is under-determined, hence the need to specify the problem for particular situations, to use free parameters or to employ extra equations to close the system. Recently Liu et al. (2007) proposed analytical expressions for the downstream

30 anisotropy as a function of the density compression ratio but only in the extreme cases of parallel and perpendicular shocks. Génot (2008) reviewed this approach and extended it to arbitrary shock angle by numerical means. Vogl et al. (2001) supplemented the RH system of equations by the mirror and firehose instabilities threshold conditions. Chao et al. (1995) proposed an expression linking upstream, downstream, and shock geometry in a

35 single equation which is finally solved numerically. Finally, to date, previous works require either a numerical solver to be employed at the end of a demanding algebraic analysis, or the knowledge of downstream parameters, or are valid close to marginal stability of specific plasma instabilities. Reinvestigating this issue we show in this paper how to express the density compression ratio as an explicit function of upstream parameters, the shock 40 geometry and the magnetic compression ratio. One motivation to choose these parameters is that magnetic measurements from spacecraft have generally less uncertainties and a better resolution than those from plasma instruments.

In the next section we present the anisotropic jump relations at a shock. In section 3 we detail the steps required to derive the analytical expression for the density compression 45 ratio, downstream anisotropy and pressure. In the last section before the conclusion we present observational tests of the methods and comparison with the earlier work of Chao et al. (1995) (in the Earth's bow shock context). An application to the heliosheath plasma state with respect to the mirror instability illustrates the sensitivity of the RH system of equations. 


\section{Anisotropic jump relations at a shock}

50 Considering a bi-Maxwellian plasma, the jump relations across a shock are (Hudson, 1970)

:

$$
\begin{gathered}
{\left[B_{n}\right]=0} \\
{\left[\rho v_{n}\right]=0} \\
{\left[v_{n} \boldsymbol{B}_{t}-\boldsymbol{v}_{t} B_{n}\right]=0} \\
{\left[P_{\perp}+\left(P_{\|}-P_{\perp}\right) \frac{B_{n}^{2}}{B^{2}}+\frac{B_{t}^{2}}{2 \mu_{0}}+\rho v_{n}^{2}\right]=0} \\
{\left[\frac{B_{n} \boldsymbol{B}_{t}}{\mu_{0}}\left(\frac{P_{\|}-P_{\perp}}{B^{2} / \mu_{0}}-1\right)+\rho v_{n} \boldsymbol{v}_{t}\right]=0} \\
{\left[\rho v_{n}\left(\frac{2 P_{\perp}}{\rho}+\frac{P_{\|}}{2 \rho}+\frac{v^{2}}{2}+\frac{B_{t}^{2}}{\mu_{0} \rho}\right)+\frac{B_{n}^{2} v_{n}}{B^{2}}\left(P_{\|}-P_{\perp}\right)-\frac{\left(\boldsymbol{B}_{t} \cdot \boldsymbol{v}_{t}\right) B_{n}}{\mu_{0}}\left(1-\frac{P_{\|}-P_{\perp}}{B^{2} / \mu_{0}}\right)\right]=0}
\end{gathered}
$$

The square brackets indicate the difference between pre-shock (upstream) and post-shock (downstream) states, $\mu_{0}$ is the permeability of the vacuum, $k$ is the Boltzmann constant, $n=\rho / m_{p}$ is the plasma density, $\boldsymbol{v}$ and $\boldsymbol{B}$ are the plasma velocity and magnetic field vec-

55 tors respectively, $P=\rho k T / m_{p}$ is the plasma pressure, and $m_{p}$ the proton mass; subscripts $t$ and $n$ denote the tangential and normal components with respect to the shock surface, and subscripts 1 and 2 in the following correspond to upstream and downstream states respectively. Without loss of generality the conservation relations are expressed in the frame where the upstream flow is parallel to the shock normal, i.e. $v_{t 1}=0$. We define the temperature

60 anisotropy by $A=T_{\perp} / T_{\|}$, the upstream Alfvén Mach number $M_{A 1}=\left(\mu_{0} \rho_{1}\right)^{1 / 2} v_{1} / B_{1}$ and $\beta_{1}=2 \mu_{0} P_{1} / B_{1}^{2}$. As mentioned previously the system of equations above is underdetermined : 6 equations, 7 unknowns $\left(=v_{n 2}, v_{t 2}, B_{n 2}, B_{t 2}, P_{\| 2}, A_{2}, \rho_{2}\right)$.

Chao et al. (1995) tackled the analytical resolution of this system with the objective of expressing the downstream anisotropy, similarly to Liu et al. (2007) and Génot (2008).

65 They obtain an expression $F$ such that $F\left(B_{2} / B_{1}, \theta_{B n}, \beta_{1}, A_{1}, \beta_{2}, A_{2}\right)=0$. For measured 
$B_{2} / B_{1}$ and $\theta_{B n}, A_{2}$ is numerically determined as a function of $\beta_{1}$ and $\beta_{2}$ (from contours plots). Applied to actually observed shock parameters this method gives good estimations of $A_{2}, r=\rho_{2} / \rho_{1}$ and $M_{A}$ (see Table 1). The drawback of the method is that (see $F$ above) the knowledge of the downstream parameter $\beta_{2}$ is required which restrains the generality

70 of the approach. Moreover a numerical solver must be finally applied. In the following we show how to remove this constraint and formulate for the first time a full analytic expression of the density compression ratio as a function of the magnetic compression ratio, the shock angle and upstream parameters only.

\section{Full analytical resolution}

75 The main challenge in solving the system of Eq. 1-6 is to eliminate the right unknown at each step. To get a full analytical solution in the end one should look for simple expressions (first or second order) of each variable.

We define $m$ which requires information of the shock itself (strength and angle)

$$
m=B_{t 2} / B_{1}=\left(\left(\frac{B_{2}}{B_{1}}\right)^{2}-\cos ^{2} \theta_{B n}\right)^{1 / 2}
$$

In our approach $m$ is considered as an input of the problem. From Eq. 3 one can express $\boldsymbol{v}_{t 2}:$

$$
\boldsymbol{v}_{t 2}=\frac{v_{n 1}}{B_{n 1}}\left(\frac{1}{r} \boldsymbol{B}_{t 2}-\boldsymbol{B}_{t 1}\right)
$$

In Eq. 4 upstream parameters are grouped together to define $C$ :

$$
C=P_{\| 1}\left(A_{1}+\left(1-A_{1}\right) \cos ^{2} \theta_{B n}\right)+\frac{B_{t 1}^{2}}{2 \mu_{0}}+\rho_{1} v_{1}^{2}\left(1-\frac{1}{r}\right)
$$

It is then possible to express $P_{\| 2}$ in terms of $C, A_{2}$ and $\boldsymbol{B}_{\mathbf{2}}$ components. Plugging this expression into Eq. 5 and making use of the expression for $\boldsymbol{v}_{t 2}$, we obtain

$$
A_{2}=\frac{E-D B_{1}^{2} \cos ^{2} \theta_{B n}}{E+D m^{2} B_{1}^{2}}
$$

with

$$
D=\left(\frac{M_{A 1}^{2}}{\cos ^{2} \theta_{B n}}-1+\frac{1}{2} \beta_{\| 1}\left(1-A_{1}\right)\right) \frac{\sin \theta_{B n}}{m}+1-\frac{M_{A 1}^{2}}{r \cos ^{2} \theta_{B n}}
$$




$$
E=\mu_{0}\left(C-\frac{m^{2} B_{1}^{2}}{2 \mu_{0}}\right)
$$

It should be recognized that $D$ is a function of $r$ therefore so is $A_{2}$. It is indeed the generalization of the expressions given in Génot (2008) for the (upstream isotropic) parallel and perpendicular shock cases. Similarly we obtain :

$$
P_{\| 2}=\frac{D m^{2} B_{1}^{2}+E}{\mu_{0}}
$$

$P_{\perp 2}$ can also be expressed by

$$
P_{\perp 2}=P_{\| 2} A_{2}=\frac{E-D B_{1}^{2} \cos ^{2} \theta_{B n}}{\mu_{0}}
$$

90 Finally plugging the expressions of $P_{\| 2}$ and $A_{2}$ (functions of $r$ ) into Eq. 6 leads to a quadratic equation in $1 / r$ whose terms are the following :

- constant term :

$$
c=-\frac{6 A_{1}+9}{8 A_{1}+4} \beta_{1}-\frac{M_{A 1}^{2}}{2 \cos ^{2} \theta_{B n}}
$$

- term in $1 / r$ :

$$
\begin{gathered}
b=\frac{15}{8 A_{1}+4}\left(A_{1}+\left(1-A_{1}\right) \cos ^{2} \theta_{B n}\right) \beta_{1}+\frac{5}{2} M_{A 1}^{2}+\frac{1}{4} m^{2}+\frac{5}{4} \sin ^{2} \theta_{B n}-\cos ^{2} \theta_{B n}+ \\
\quad \frac{\sin \theta_{B n}}{m} \times\left(\frac{3}{2} m^{2}-\cos ^{2} \theta_{B n}\right) \times\left(\frac{M_{A 1}^{2}}{\cos ^{2} \theta_{B n}}-1+\frac{3\left(1-A_{1}\right)}{4 A_{1}+2} \beta_{1}\right)
\end{gathered}
$$

- term in $1 / r^{2}$ :

$$
a=-M_{A 1}^{2}\left(1+\frac{m^{2}}{\cos ^{2} \theta_{B n}}\right)
$$

95 We used $3 \beta_{1}=\left(1+2 A_{1}\right) \beta_{\| 1}$ in Eq. 15 and Eq. 16. By solving the quadratic equation $a / r^{2}+b / r+c=0$, the compression ratio can be explicitly obtained as a function of upstream parameters, the shock angle and the magnetic compression ratio. The physical solution is : 


$$
r=\frac{2 a}{-b+\sqrt{b^{2}-4 a c}}=F\left(\frac{B_{2}}{B_{1}}, \theta_{B n}, A_{1}, M_{A 1}, \beta_{1}\right)
$$

For exactly perpendicular shock $\left(r=B_{2} / B_{1}=m\right)$ the method diverges (because of the terms in $\left.1 / \cos \theta_{B n}\right)$. For exactly parallel shock $\left(B_{1}=B_{2}, m=0\right)$ the method is not appropriate. Simplified approaches (Liu et al., 2007; Génot, 2008) must therefore be used for $\theta_{B n}=0^{\circ}$ and $\theta_{B n}=90^{\circ}$. However the present approach gives consistent results even for angle very close to $90^{\circ}$. For a given set of upstream parameters all oblique shocks are not physical and the positivity of the discriminant $\left(b^{2}-4 a c\right)$ will determine their validity.

105 Knowing $r, D$ (Eq. 11) and $E$ (Eq. 12) are fully determined and so are the downstream anisotropy and pressure. Explicit solutions are too lengthy to be written but are straightforward from Eq. 10 and Eq. 13.

\section{Observational tests}

\subsection{Comparison with Earth's bow shock data}

110 The applicability of R-H jump conditions to observed shocks has been verified (for instance Winterhalter et al. (1984)). To validate the present approach we use six bow shocks crossings referenced in Chao et al. (1995) (see Table 1). They all correspond to low Mach number solar wind conditions. Alfvén Mach numbers in Table 1 are computed from Eq. 12 in Chao et al. (1995). The last three columns of Table 1 display the density compression ratio as it is

115 observed, from our Eq. 18 and from equations of Chao et al. (1995) respectively. For a given shock, differences between the three values are very small. First, our (direct) method gives results very close to those obtained by the method of Chao et al. (1995). Slight discrepancies may come from our use of calculated $M_{A}$ (round values instead of exact). Second, our calculated ratios agree very well with observed values.

\subsection{Comparison with Termination Shock data}

In the following we illustrate the sensitivity of downstream conditions (mainly the pressure and anisotropy) to the input parameters. On 2004, December 16 Voyager 1 crossed the solar wind termination shock at $94 \mathrm{AU}$ and entered the heliosheath. Magnetic field measurements revealed similarities with planetary magnetosheath : fluctuations resembling holes and peaks associated with the mirror instability were observed (Burlaga et al., 2006; Génot et al., 
2009). This led several authors to investigate whether the heliosheath plasma was unstable with respect to this instability, i.e. wether the mirror condition $C_{M}=\beta_{\perp 2}\left(A_{2}-1\right)>1$ was fulfilled. However due to the lack of plasma data, simulations were performed to infer the upstream plasma conditions $\left(M_{A 1}=16.3\right.$ and $\beta_{1}=32.8$ (Whang et al., 2004)) and jump conditions were used to determine the downstream ones (Liu et al., 2007; Génot, 2008). For this crossing the magnetic jump is observed to be $B_{2} / B_{1}=3$. Assuming isotropic upstream solar wind and an exactly perpendicular shock (case 1 in Table 2) gives an unstable heliosheath plasma. For the same shock a slightly anisotropic solar wind gives an even more unstable heliosheath plasma (case 2 equivalent to the analysis of Liu et al. (2007)).

135 However it has been inferred for this crossing that $\theta_{B n}=86^{\circ}$ rather than $\theta_{B n}=90^{\circ}$. It can be seen from case 3 and 4 that this situation corresponds to a stable heliosheath plasma in contradiction with previous conclusions. Case 5 completes the demonstration showing the extreme sensitivity of the RH system : the magnetic ratio is decreased to $B_{2} / B_{1}=2.99$ to recover an unstable plasma. This analysis shows that one must be very cautious with results

140 obtained from the RH jump relations. It is necessary to precisely evaluate the error bars on downstream parameters from the uncertainties on input quantities (to be developed in a forthcoming paper). Due to this sensitivity the upstream wave turbulence may also have important consequences on the downstream solutions of the RH system. The way MHD fluctuations may affect the shock properties has been analyzed, for instance, in Lerche et al.

145 (2000) for isotropic plasma.

\section{Conclusion}

The analysis developed in this work is intended to complete general studies on anisotropic MHD shocks by giving, for the first time, a full analytic expression of the density compression ratio as a function of the upstream parameters and shock angle and strength. It has been validated by comparison with another method and observations in different astrophysical contexts. Such compact formula may be used to easily compute downstream parameters when only magnetic measurements are available and when upstream parameters can be inferred (when plasma data are absent, in the case of Voyager 1 for instance). It is also possible to analyze the sensitivity of the results to uncertainties in the inputs and to propose error bars. This works could pave the way to further analytical analysis of more complex Rankine-Hugoniot systems, taking into account the waves and/or turbulence and/or heat 
flow (Chao and Goldstein, 1972). 


\section{References}

Burlaga L. F., Ness N. F. , and Acũna M. H.: Trains of magnetic holes and magnetic humps in the heliosheath, Geophys. Res. Lett., 33, L21106, doi:10.1029/2006GL027276, 2006.

Chao, J. K., and Goldstein, B.: Modification of the Rankine-Hugoniot Relations for Shocks in Space, J. Geophys. Res., 77, 28, 1972.

Chao, J. K., Zhang, X. X., and Song, P.: Derivation of temperature anisotropy from shock jump relations: Theory and observations, Geophys. Res. Lett., 22, 17, 1995.

165 Génot, V., Mirror and firehose instabilities in the heliosheath, The Astrophysical Journal, 687, L119L122, 2008.

Génot, V., Budnik, E., Hellinger P., Passot T., Belmont G., Travnicek P., Sulem P.-L., Lucek, E., and Dandouras, I.: Mirror structures above and below the linear instability threshold : Cluster observations, fluid model and hybrid simulations, Ann. Geophys., 27, 601-615, 2009.

170 Hudson, P. D.: Discontinuities in an anisotropic plasma and their identification in the solar wind, Planet. Space Sci., 18, 1611, 1970.

Lerche, I., Pohl, M., and Schlickeiser, R.: Turbulent adiabatic shock waves and diffusive particle acceleration, J. Plasma Phys., 64, 459-474, 2000.

Liu, Y., Richardson, J. D., Belcher, J. W., and Kasper, J. C.: Temperature anisotropy in a shocked 175 plasma: mirror-mode instabilities in the heliosheath, The Astrophysical Journal, 659, L65, 2007.

Siewert, M., and Fahr, H.-J., A Boltzmann-kinetical description of an MHD shock with arbitrary field inclination, Astron. Astrophys., 485, 327-336, 2008.

Vogl, D. F., Biernat, H. K., Erkaev, N. V., Farrugia, C. J., and Mühlbachler, S.: Jump conditions for pressure anisotrophy and comparison with the Earth's bow shock, Nonlin. Proc. Geophys., 8, 3, 1802001.

Whang, Y. C., Burlaga L. F., Wang Y.-M.,

Sheeley Jr. N. R.: The termination shock near $35^{\circ}$ latitude, Geophys. Res. Lett., 31, L03805, doi:10.1029/2003GL018679, 2004.

Winterhalter, D., Kivelson M. G., Walker R. J., and Russell C. T.: The MHD Rankine-Hugoniot jump conditions and the terrestrial bow shock - A statistical comparison, Advances in Space Research, vol. 4, no. 2-3, p. 287-292, 1984. 
Tables

\begin{tabular}{|c|c|c|c|c|c|c|c|}
\hline Event & $B_{2} / B_{1}$ & $\theta_{B n}\left({ }^{\circ}\right)$ & $\beta_{1}$ & $M_{A 1}$ & $r$ (obs.) & $r$ (this method) & $r$ (Chao et al. (1995)) \\
\hline 1 & 2.65 & 71.4 & 0.17 & 3.3 & 2.70 & 2.71 & 2.70 \\
2 & 2.78 & 79.9 & 0.15 & 3.8 & 2.70 & 2.80 & 2.78 \\
3 & 2.72 & 86.1 & 0.12 & 3.5 & 2.63 & 2.72 & 2.70 \\
4 & 2.25 & 65.5 & 0.17 & 2.4 & 2.27 & 2.32 & 2.27 \\
5 & 2.18 & 64.5 & 0.16 & 2.2 & 2.33 & 2.25 & 2.27 \\
6 & 1.99 & 53.7 & 0.10 & 2.0 & 2.13 & 2.08 & 2.08 \\
\hline
\end{tabular}

Table 1. Six Earth's bow shock crossings referenced in Chao et al. (1995) and associated parameters : observed magnetic amplitude ratio, shock angle and upstream $\beta$, calculated upstream Alfvén Mach number (see text) and observed density compression ratio; the last two columns are the calculated density compression ratio obtained from Eq. 18 and from the method of Chao et al. (1995) respectively.

\begin{tabular}{|c|c|c|c|c|}
\hline Case & $\theta_{B n}\left(^{\circ}\right)$ & $A_{1}$ & $r$ & $C_{M}=\beta_{\perp 2}\left(A_{2}-1\right)$ \\
\hline 1 & 90 & 1 & 3 & 1.17 \\
2 & 90 & 0.94 & 3 & 1.25 \\
3 & 86 & 1 & 3.006 & 0.83 \\
4 & 86 & 0.94 & 3.006 & 0.92 \\
5 & 86 & 0.94 & 2.996 & 1.48 \\
\hline
\end{tabular}

Table 2. Observed and calculated parameters for the solar wind Termination Shock crossed by Voyager 1 in 2004. The shock angle (row 2) and upstream anisotropy (row 3) are slightly varied to reveal the sensitivity of the RH system of equations : this is expressed in the large variations of the mirror mode criterion $C_{M}$ (row 5) while the density compression ratio (row 4) remains almost constant. For this crossing it was inferred that $M_{A 1}=16.3$ and $\beta_{1}=32.8$ (Whang et al., 2004) and observed that $B_{2} / B_{1}=3$ (Burlaga et al., 2006). This later value is used for cases 1-4 whereas $B_{2} / B_{1}=2.99$ is used in case 5 . 